\title{
Por que amamentar?: Uma revisão de literatura
}

\author{
Why breastfeed?: A literature review \\ ¿Por qué amamantar?: Una revisión de la literatura
}

Geovanna Renaissa Ferreira Caldas

ORCID: https://orcid.org/0000-0001-9820-309X

Centro Universitário de Juazeiro do Norte, Brasil E-mail: geovannacaldas@ hotmail.com

Maria Gabriela dos Santos

ORCID: https://orcid.org/0000-0002-9443-0815

Hospital da Mulher Dr. ${ }^{a}$ Nise da Silveira, Brasil E-mail: mariagabi111@hotmail.com

Polyane Salgado Areia

ORCID: https://orcid.org/0000-0003-2360-7625 Instituto de Ensino Superior Franciscano, Brasil E-mail: pollyanesalgado18@gmail.com

Samara Atanielly Rocha

ORCID: https://orcid.org/0000-0002-5622-9280

Faculdade de Saúde e Humanidades Ibituruna, Brasil E-mail: samaraatanielly@ outlook.com Eva Natalina Ferreira Costa

ORCID: https://orcid.org/0000-0003-2673-6967 Universidade Federal do Estado do Rio de Janeiro, Brasil E-mail: evacostaferreira@gmail.com

Flávia Martins Branco

ORCID: https://orcid.org/0000-0003-3484-8555 Universidade Federal do Estado do Rio de Janeiro, Brasil E-mail: flaviabrancoenf@gmail.com

Dayane Martins da Silva Campos

ORCID: https://orcid.org/0000-0002-0193-4417 Universidade Federal do Estado do Rio de Janeiro, Brasil E-mail: campos_dayane@hotmail.com

Marcos Soares de Lima

ORCID: https://orcid.org/0000-0002-0721-5771 Universidade de Pernambuco, Brasil E-mail: marcos55soares@hotmail.com

Karinne Naziazena da Silva Monteiro ORCID: https://orcid.org/0000-0003-0014-6790 Centro Universitário UNIFG, Brasil E-mail: karinnemonteiro16@gmail.com

Evelyn Azevedo Bispo da Silva ORCID: https://orcid.org/0000-0002-9400-7726 Universidade Católica de Pernambuco, Brasil E-mail: evelynazev@gmail.com

Samuel Melo Ribeiro

ORCID: https://orcid.org/0000-0003-2582-7883

Faculdade de Saúde e Ecologia Humana, Brasil

E-mail: samuelmeloribeiro@hotmail.com

Bianca Rodrigues Tavares

ORCID: https://orcid.org/0000-0002-7340-7309

Faculdade de Saúde e Ecologia Humana, Brasil

E-mail: biancatavares20@yahoo.com.br Ana Carolina Pinto da Silva

ORCID: https://orcid.org/0000-0002-9965-1772 Universidade Federal da Bahia, Brasil

E-mail: ana-carolina_pinto@hotmail.com

Miller Fontes Brandão

ORCID: https://orcid.org/0000-0002-4525-1845 Universidade Federal da Bahia, Brasil

E-mail: miller_brandao_100@hotmail.com Jackson da Silva

ORCID: https://orcid.org/0000-0002-5373-9699 Centro Universitário UNIFBV, Brasil E-mail: jacksonsilva10@bol.com.br 


\title{
Vilma Regina Ferreira Rodrigues \\ ORCID: https://orcid.org/0000-0002-8976-5477 Universidade do Estado do Pará, Brasil E-mail: enf.vilmarodrigues@gmail.com \\ Márcia Audielen Ferreira Rodrigues ORCID: https://orcid.org/0000-0001-8964-0482 Faculdade Pitágoras de Imperatriz, Brasil E-mail: marcia.audiellen@gmail.com \\ Maria Elisa Regina Benjamin de Moura ORCID: https://orcid.org/0000-0003-3145-4307 Centro Universitário de Juazeiro do Norte, Brasil E-mail: elisareg@gmail.com \\ Dayse Christina Rodrigues Pereira Luz ORCID: https://orcid.org/0000-0002-5719-3574 Faculdade de Medicina do ABC, Brasil E-mail: dayseluz.dcrp@gmail.com
}

\begin{abstract}
Resumo
O objetivo do estudo foi evidenciar os benefícios da amamentação para recém-nascidos prematuros. Trata-se de uma revisão integrativa da literatura, onde utilizou-se a Literatura Latino-Americana e do Caribe em Ciências da Saúde, Scientific Electronic Library Online e Medical Literature Analysis and Retrievel System Online, com os Descritores em Ciências da Saúde (DECS) "Amamentação AND Crescimento e Desenvolvimento AND Prematuro". Após aplicação dos critérios de inclusão e exclusão, utilizou-se o protocolo PRISMA para elucidação da seleção e uma tabela para evidenciar os 13 estudos escolhidos para compor a revisão. O prematuro deve receber como primeira e se possível, única escolha, o leite materno, pois este é suficiente para as necessidades do bebê, possuindo ainda, várias qualidades imunológicas, que irão auxiliar no crescimento, desenvolvimento e na imaturidade, proporciona ainda, diversos benefícios para mãe e filho, atuando sobre a saúde física, biológica e mental, mas transparece dificuldades enfrentadas para poder usufruir desses benefícios, como a amamentação exclusiva não ser frequentemente alcançada. Conclui-se que a amamentação deve ser muito bem orientada e estimulada para a sua implementação, pois acarreta benefícios para a mãe e para o bebê, auxiliando na bem-estar e na saúde física e mental dos dois.
\end{abstract}

Palavras-chave: Amamentação; Aleitamento materno; Recém nascido prematuro; Crescimento e desenvolvimento.

\begin{abstract}
The aim of the study was to highlight the benefits of breastfeeding for premature newborns. This is an integrative literature review, using Latin American and Caribbean Literature in Health Sciences, Scientific Electronic Library Online and Medical Literature Analysis and Retrievel System Online, with Health Sciences Descriptors (DECS) "Breastfeeding AND Growth and Development AND Premature". After applying the inclusion and exclusion criteria, the PRISMA protocol was used to elucidate the selection and a table to highlight the 13 studies chosen to compose the review. Premature infants should receive breast milk as the first and, if possible, the only choice, as this is sufficient for the baby's needs, as well as having several immunological qualities, which will assist in growth, development and immaturity, providing several benefits as well. for mother and child, acting on physical, biological and mental health, but there are difficulties faced in order to enjoy these benefits, as exclusive breastfeeding is not often achieved. It is concluded that breastfeeding must be very well oriented and stimulated for its implementation, as it brings benefits to the mother and the baby, aiding in the well-being and physical and mental health of both.
\end{abstract}

Keywords: Breast-feeding; Breastfeeding; Premature newborn; Growth and development.

\section{Resumen}

El objetivo del estudio fue destacar los beneficios de la lactancia materna para los recién nacidos prematuros. Se trata de una revisión de literatura integradora, utilizando Literatura Latinoamericana y del Caribe en Ciencias de la Salud, Biblioteca Electrónica Científica en Línea y Sistema de Análisis y Recuperación de Literatura Médica en Línea, con Descriptores de Ciencias de la Salud (DECS) "Lactancia Materna Y Crecimiento y Desarrollo Y Prematuro". Después de aplicar los criterios de inclusión y exclusión, se utilizó el protocolo PRISMA para dilucidar la selección y una tabla para resaltar los 13 estudios elegidos para componer la revisión. El bebé prematuro debe recibir la leche materna como primera y, si es posible, única opción, ya que esta es suficiente para las necesidades del bebé, además de poseer varias cualidades inmunológicas, las cuales ayudarán en el crecimiento, desarrollo e inmadurez, brindando varios beneficios para el bebé. madre e hijo, actuando sobre la salud física, biológica y mental, pero existen dificultades para poder disfrutar de estos beneficios, ya que no es frecuente que se logre la lactancia materna exclusiva. Se concluye que la lactancia materna debe estar muy bien orientada y estimulada para su implementación, ya que aporta beneficios a la madre y al bebé, contribuyendo al bienestar y la salud física y mental de ambos.

Palabras clave: Amamantamiento; Amamantamiento; Recién nacido prematuro; Crecimiento y desarrollo. 


\section{Introdução}

Segundo o Caderno de Atenção Básica no 23 (CAB 23) do Ministério da Saúde, a amamentação possui diversos benefícios, proporcionando o estímulo do vínculo entre mãe e filho, auxiliando na parte nutricional da criança, onde estará diretamente relacionada com o crescimento e o desenvolvimento físico, motor e cognitivo da criança, evitando mortes ou doenças. Em relação a mãe, pode incluir implicações na parte física e mental, além de proteção contra alguns tipos de câncer, ser um método anticoncepcional e gerar menos custos. Garantindo então, melhores condições de vida para mãe e filho (Ministério da Saúde, 2015).

A Organização mundial de saúde (OMS) orienta que a criança seja amamentada de forma exclusiva até os 6 meses, não necessitando nem de água, pois o leite materno a única necessidade do recém-nascido (RN), composto de água, carboidratos, proteínas e lipídeos. Esse tipo de informação deve ser repassada as mães desde o planejamento familiar, durante todas as consultas de pré-natal e reforçado nas consultas de puericultura, no intuito de evitar o desmame precoce e de sanar as dúvidas e angústias das puérperas, visto que muitas consideram o leite "fraco" ou insuficiente" (Alves; Oliveira; Rito, 2018 \& Rocha et al., 2020).

A amamentação é mais complexa de ser iniciada e sustentada em crianças prematuras, isso ocorre devido dificuldades sucedidas da imaturidade nas capacidades de sucção e deglutição, isso faz com que os RN prematuros cansem rápido da mamada, sendo de menor intensidade em relação a duração, mas também menor tempo de intervalo entre uma e outra (Spezzia, 2020).

A prematuridade pode ser definida como o nascimento de um feto com menos de 37 semanas de Idade Gestacional (IG), podendo ser classificado em tardio, moderado e extremo. Ela configura-se como um dos maiores problemas enfrentados pela saúde, sendo de caráter mundial, possuindo uma origem multicausal e afetando diretamente a saúde da criança, originando patologias e dificuldades em todas os aspectos, carecendo de maior atenção e podendo evoluir até ao óbito (Gomes et al., 2020).

Dentre as patologias que os prematuros são mais susceptíveis de vir a desenvolver, destacam-se problemas no sistema imunológico, doenças do sistema respiratório, doenças do sistema cardiovascular problemas gástricos e intestinais, entre outros (Rosa et al., 2020).

A prematuridade segue acompanhada com uma imaturidade fisiológica, advinda da falta de crescimento e desenvolvimento, essa imaturidade gera dificuldades para a criança ou atraso em alguns reflexos, como o reflexo de busca e sucção do leite materno. Tal atraso afeta diretamente a amamentação, pois durante a suç̧ão é liberado a ocitocina, hormônio que estimula a produção leite, ou seja para que o corpo entenda que deve produzir leite materno, é necessário que ocorra a sucção como um estímulo para tal, dificultado pela imaturidade sucedida da criança prematura (Monteiro et al., 2020 \& Vitolo, 2014).

Destaca-se a relevância da seguinte pesquisa por abordar importantes informações sobre a saúde materno-infantil, principalmente para a área neonatal e pediátrica, a qual pode proporcionar elaborações de futuras estratégias voltadas para o assunto ou de pesquisas relacionadas ao abordado, em busca da melhoria na qualidade da assistência para mãe e filho, além ainda, de apresentar um tema de grande relevância, pois é um grande problema vivenciado pela saúde mundial. Sendo assim, surgiu a seguinte questão norteadora: Qual a influência da amamentação no crescimento e desenvolvimento de recém-nascidos prematuros?

Em virtude disso, o objetivo do estudo foi evidenciar os benefícios da amamentação para recém-nascidos prematuros.

\section{Metodologia}

Trata-se de uma revisão integrativa da literatura, segundo Gonçalves (2019), deve-se iniciar o processo com a 
definição do tema, seguido por levantamento de artigos na literatura, os quais devem ser lidos e escolhidos para fazer parte da revisão e montagem do quadro nos resultados e exemplificação nas discussões, garantindo um bom entendimento sobre o assunto abordado pela revisão.

O presente documento baseia-se em estudos qualitativos. Na pesquisa qualitativa o próprio pesquisador é o principal instrumento durante todo o processo, considerando relevante a interpretação do pesquisador/autor, através de respostas do público obtidas por meio de perguntas abertas, dando liberdade para o entrevista comentar e dar sua opinião sobre, a partir dai, o escrito atua intercalando essas respostas e implementando opiniões, analisando por um processo indutivo (Pereira et al., 2018).

Para alcance dos estudos utilizados, efetivou-se uma investigação na plataforma Biblioteca Virtual em Saúde (BVS), a qual possui diversas bases de dados, entre elas, foram selecionadas a Literatura Latino-Americana e do Caribe em Ciências da Saúde (LILACS), Scientific Electronic Library Online (SciELO) e Medical Literature Analysis and Retrievel System Online (MEDLINE). Para pesquisa utilizou-se o emprego dos Descritores em Ciências da Saúde (DECS) "Amamentação, Crescimento e Desenvolvimento e Prematuro", agregados ao operador booleano "AND", aplicando-se na seguinte sequência: "Amamentação AND Crescimento e Desenvolvimento AND Prematuro".

Em relação aos critérios de inclusão e exclusão, menciona-se como de critérios de inclusão: artigos originais, artigos que se encontrassem disponíveis completos na íntegra de forma gratuita, com publicação nos idiomas português, espanhol e/ou inglês, que abordassem o tema do estudo, permitindo responder à questão norteadora, cita-se ainda, que tivessem sido publicados nos últimos 5 anos (2016 a 2021). Como critérios de exclusão destacam-se: artigos repetidos em bases diferentes, outras revisões de literatura ou sistemáticas, resumos simples, resumos expandidos e/ou estudos que não apresentassem uma devida conclusão.

\section{Resultados}

Com base nas buscas efetivadas, alcançou-se 147 artigos na BVS (Incluindo LILACS e MEDLINE) e 2 artigos na SciELO, totalizando 149 artigos, que após aplicação dos critérios de inclusão e exclusão relatados anteriormente, restaram 72 documentos, dos quais, 13 estudos foram utilizados para a confecção da seguinte revisão, sendo esses, 11 em língua inglesa, 2 em língua portuguesa, 12 artigos retirados na base MEDLINE e 1 artigo retirado na base SCIELO.

Para esclarecimento de todo método de busca e escolha dos estudos empregados, utilizou-se um fluxograma baseado no protocolo PRISMA, ilustrado na Figura 1. 
Figura1 - Fluxograma de escolha dos estudos.

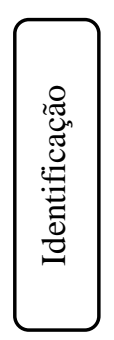

\section{Ao realizar pesquisa dos DeCS na} base de dados LILACS $(\mathrm{n}=27)$
Ao realizar pesquisa dos DeCS na base de dados MEDLINE ( $\mathrm{n}$ $=120$ )
Ao realizar pesquisa dos DeCS na base de dados SCIELO $(n=2)$

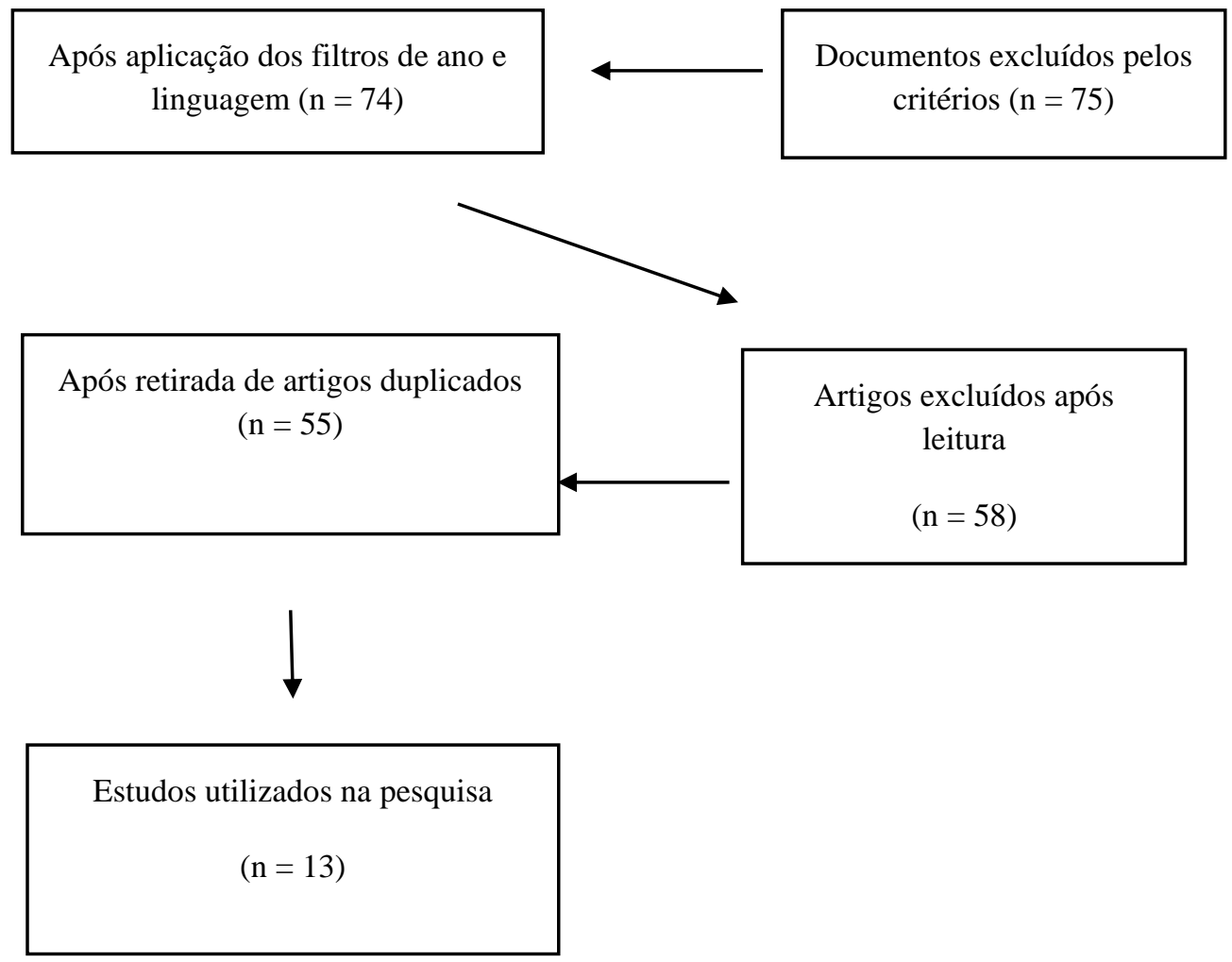

Fonte: Protocolo PRISMA

Posteriormente, realizou-se a leitura dos artigos elegidos, as informações colhidos foram inseridos em uma tabela (Quadro 1), extraindo-se os seguintes elementos: autor, ano, título, objetivo e perfil da amostra, usados para a organização e demonstração. 
Quadro 1 - Estudos utilizados para a revisão.

\begin{tabular}{|c|c|c|c|}
\hline AUTORES E ANO & TITULO & OBJETIVO & PERFIL DA AMOSTRA \\
\hline $\begin{array}{l}\text { Bezerra, M.J. et al., } \\
2017 .\end{array}$ & $\begin{array}{l}\text { Percepção de mães de } \\
\text { recém-nascidos } \\
\text { prematuros } \\
\text { hospitalizados acerca da } \\
\text { amamentação. }\end{array}$ & $\begin{array}{l}\text { Compreender como as mães } \\
\text { percebem o processo de } \\
\text { amamentação de seu filho } \\
\text { prematuro hospitalizado na } \\
\text { Unidade de Terapia Intensiva } \\
\text { Neonatal. }\end{array}$ & $\begin{array}{l}\text { Realizado com } 8 \text { mães em uma } \\
\text { cidade do interior do Ceará, Brasil. } \\
\text { Os dados foram coletados por meio } \\
\text { de entrevista semiestruturada entre } \\
\text { agosto e setembro de } 2015 \text {. }\end{array}$ \\
\hline $\begin{array}{l}\text { Nunes, C.R.N. et al., } \\
2017 .\end{array}$ & $\begin{array}{l}\text { Relação da duração da } \\
\text { posição canguru e } \\
\text { interação mãe-filho pré- } \\
\text { termo na alta hospitalar. }\end{array}$ & $\begin{array}{l}\text { Analisar a influência da duração } \\
\text { da Posição Canguru nas } \\
\text { interações iniciais da díade mãe- } \\
\text { filho pré-termo. }\end{array}$ & $\begin{array}{l}\text { Todos os recém-nascidos elegíveis } \\
\text { (idade gestacional entre } 28 \text { e } 32 \\
\text { semanas e peso ao nascimento entre } \\
1000 \text { e } 1800 \mathrm{~g} \text { ) foram incluídos no } \\
\text { período de } 11 \text { de junho a } 31 \text { de } \\
\text { setembro de } 2014 \text {. }\end{array}$ \\
\hline $\begin{array}{l}\text { Fonseca, L.T. et al., } \\
2018 .\end{array}$ & $\begin{array}{l}\text { Association between } \\
\text { human breast milk and } \\
\text { retinopathy } \\
\text { prematurity. }\end{array}$ & $\begin{array}{l}\text { Avaliar o possível efeito } \\
\text { protetor do leite materno contra } \\
\text { a retinopatia da prematuridade }\end{array}$ & $\begin{array}{l}\text { Recém-nascidos com peso ao } \\
\text { nascer }<1500 \mathrm{~g} \text { ou idade } \\
\text { gestacional }<32 \text { semanas, ou ambas, } \\
\text { nascidos entre janeiro de } 2011 \text { e } \\
\text { outubro de } 2014 \text { e internados nas } \\
\text { primeiras } 24 \text { horas de vida na } \\
\text { Unidade de Terapia Intensiva } \\
\text { Neonatal do Hospital Criança. }\end{array}$ \\
\hline Luz, L.S. et al., 2018. & $\begin{array}{l}\text { Predictive factors of the } \\
\text { interruption of exclusive } \\
\text { breastfeeding in } \\
\text { premature infants: a } \\
\text { prospective cohort. }\end{array}$ & $\begin{array}{l}\text { Avaliar a incidência do } \\
\text { aleitamento materno exclusivo e } \\
\text { os fatores de risco associados à } \\
\text { interrupção de aleitamento } \\
\text { materno exclusivo } r \text { em } \\
\text { prematuros após a alta } \\
\text { hospitalar. }\end{array}$ & $\begin{array}{l}113 \text { prematuros em unidade } \\
\text { neonatal, e acompanhados entre } 7 \text { e } \\
15 \text { dias após a alta hospitalar. }\end{array}$ \\
\hline $\begin{array}{l}\text { Toftlund, L.H. et al., } \\
2018 .\end{array}$ & $\begin{array}{l}\text { Catch-Up Growth, Rapid } \\
\text { Weight Growth, and } \\
\text { Continuous Growth from } \\
\text { Birth to } 6 \text { Years of Age } \\
\text { in Very-Preterm-Born } \\
\text { Children. }\end{array}$ & $\begin{array}{l}\text { Investigar como diferentes tipos } \\
\text { de nutrição pós-alta afetam o } \\
\text { crescimento até os } 6 \text { anos de } \\
\text { idade em bebês muito } \\
\text { prematuros. }\end{array}$ & $\begin{array}{l}320 \text { bebês prematuros muito e } \\
\text { extremamente prematuros, com IG } \\
\leq 32 \text { semanas de } 4 \text { unidades } \\
\text { neonatais dinamarquesas, que } \\
\text { estavam em pós- nutrição de alta. }\end{array}$ \\
\hline Tonkin, E. et al., 2018. & $\begin{array}{l}\text { Dietary Protein Intake, } \\
\text { Breast Feeding and } \\
\text { Growth in Human Milk } \\
\text { Fed Preterm Infants. }\end{array}$ & $\begin{array}{l}\text { Descrever a ingestão real de } \\
\text { proteína e energia de bebês } \\
\text { prematuros alimentados com } 2 \\
\text { níveis diferentes de proteína } \\
\text { durante sua admissão neonatal. }\end{array}$ & $\begin{array}{l}98 \text { bebês com <31 semanas de } \\
\text { gestação, investigando dois níveis } \\
\text { de fortificação de proteína, }\end{array}$ \\
\hline
\end{tabular}




\begin{tabular}{|c|c|c|c|}
\hline Blesa, M. et al., 2019. & $\begin{array}{l}\text { Early breast milk } \\
\text { exposure modifies brain } \\
\text { connectivity in preterm } \\
\text { infants. }\end{array}$ & $\begin{array}{l}\text { Testamos a hipótese de que a } \\
\text { exposição ao leite materno está } \\
\text { associada a melhores } \\
\text { marcadores de desenvolvimento } \\
\text { cerebral e conectividade em } \\
\text { bebês prematuros com idade } \\
\text { equivalente a termo. }\end{array}$ & $\begin{array}{l}\text { Bebês prematuros nascidos com } \leq \\
33,0 \text { semanas que receberam } \\
\text { atendimento na Royal Infirmary de } \\
\text { Edimburgo e tiveram ressonância } \\
\text { magnética do cérebro. }\end{array}$ \\
\hline $\begin{array}{l}\text { Congiu, M. et al., } \\
2019 .\end{array}$ & $\begin{array}{l}\text { Breast Milk for Preterm } \\
\text { Multiples: More } \\
\text { Proteins, Less Lactose. }\end{array}$ & $\begin{array}{l}\text { Investigar o papel de alguns } \\
\text { fatores adicionais que } \\
\text { potencialmente influenciam as } \\
\text { propriedades benéficas do leite } \\
\text { materno em gestações múltiplas. }\end{array}$ & $\begin{array}{l}51 \text { recém nascidos ( } 25 \text { meninos e } \\
26 \text { meninas), com IG média de } \\
28,92 \pm 2,5 \text { semanas e PN médio de } \\
1076,62 \pm 318,45 \mathrm{~g} \text { em uma UTI } \\
\text { Neonatal. }\end{array}$ \\
\hline $\begin{array}{l}\text { Ericson, J., Palmér, L., } \\
2019 .\end{array}$ & $\begin{array}{l}\text { Mothers of preterm } \\
\text { infants' experiences of } \\
\text { breastfeeding support in } \\
\text { the first } 12 \text { months after } \\
\text { birth: A qualitative } \\
\text { study. }\end{array}$ & $\begin{array}{l}\text { Descrever como mães de bebês } \\
\text { prematuros na Suécia vivenciam } \\
\text { o apoio à amamentação durante } \\
\text { os primeiros } 12 \text { meses após o } \\
\text { nascimento. }\end{array}$ & $\begin{array}{l}\text { Utilizou dados de } 151 \text { mães de } \\
\text { questionários com perguntas } \\
\text { abertas e entrevistas telefônicas. Os } \\
\text { dados foram analisados por uma } \\
\text { rede temática indutiva com } \\
\text { abordagem hermenêutica. }\end{array}$ \\
\hline $\begin{array}{l}\text { Halleux, V. et al., } \\
2019 .\end{array}$ & $\begin{array}{l}\text { Growth Benefits of Own } \\
\text { Mother's Milk in Preterm } \\
\text { Infants Fed Daily } \\
\text { Individualized Fortified } \\
\text { Human Milk. }\end{array}$ & $\begin{array}{l}\text { Avaliar o crescimento em bebês } \\
\text { MBPN alimentados com HM } \\
\text { fortificado individualizado com } \\
\text { OMM predominante }(\geq 75 \%) \text { ou } \\
\text { DM predominante }(\geq 75 \%) \text {. }\end{array}$ & $\begin{array}{lrr}\text { Prematuros nascidos com } & <32 \\
\text { semanas de gestação } & \text { (IG) } \\
\text { internados na UTIN da } & \\
\text { Universidade de Liège, Bélgica. }\end{array}$ \\
\hline $\begin{array}{l}\text { Marino, L.V. et al., } \\
2019 .\end{array}$ & $\begin{array}{l}\text { Home use of breast milk } \\
\text { fortifier to promote } \\
\text { postdischarge growth } \\
\text { and breast feeding in } \\
\text { preterm infants: a quality } \\
\text { improvement project. }\end{array}$ & $\begin{array}{l}\text { Melhorar o crescimento em } \\
\text { bebês prematuros amamentados } \\
\text { exclusivamente }(\leq 1,8 \mathrm{~kg} \text { ao } \\
\text { nascer }) \text { após a alta, introduzindo } \\
\text { o uso rotineiro de BMF após a } \\
\text { alta. }\end{array}$ & $\begin{array}{l}\text { Bebês prematuros amamentados } \\
\text { exclusivamente }(\leq 1,8 \mathrm{~kg} \text { ao nascer }) \\
\text { na Unidade Neonatal de } \\
\text { Southampton. }\end{array}$ \\
\hline $\begin{array}{l}\text { McKinney C.M. et al., } \\
2019 .\end{array}$ & $\begin{array}{l}\text { A randomized crossover } \\
\text { trial comparing the Nifty } \\
\text { cup to a medicine cup in } \\
\text { preterm infants who have } \\
\text { difficulty breastfeeding } \\
\text { at Komfo Anokye } \\
\text { Teaching Hospital } \\
\text { (KATH) in Kumasi, } \\
\text { Ghana. }\end{array}$ & $\begin{array}{l}\text { Avaliar o copo Nifty, um novo } \\
\text { copo de alimentação projetado } \\
\text { especificamente para bebês com } \\
\text { dificuldades de amamentação. }\end{array}$ & $\begin{array}{l}200 \text { mães e } 237 \text { bebês prematuros } \\
\text { com dificuldades de amamentação } \\
\text { indicados para alimentação com } \\
\text { copo no Hospital Escola Komfo } \\
\text { Anokye. }\end{array}$ \\
\hline $\begin{array}{l}\text { Nandakumar, A. et al., } \\
2020 .\end{array}$ & $\begin{array}{l}\text { Exclusive Breast Milk } \\
\text { vs. Hybrid Milk Feeding }\end{array}$ & $\begin{array}{l}\text { Comparar o tempo para atingir } \\
\text { FEF em bebês prematuros }\end{array}$ & $\begin{array}{l}\text { Neonatos prematuros nascidos entre } \\
27 \text { e } 32 \text { semanas de gestação e peso }\end{array}$ \\
\hline
\end{tabular}




\begin{tabular}{|l|l|l|l|l|}
\hline & for Preterm Babies-A & alimentados com "leite materno & ao nascer <1.500 g em uma UTI de \\
Randomized Controlled & sozinho (MM)" vs. bebês & um hospital da Índia. \\
Trial Comparing Time to & prematuros alimentados com & \\
Full Feeds. & "leite híbrido alimentado com & \\
& & leite materno suplementado com & \\
& leite artificial (HF)". & \\
\hline
\end{tabular}

Fonte: Autores.

\section{Discussão}

O aleitamento materno proporciona diversos benefícios para mãe e filho, atuando sobre a saúde física, biológica e mental. Sabendo da sua importância, os profissionais da saúde devem estimular a amamentação exclusiva até os 6 meses, especialmente para mães com filhos prematuros, pois alguns desses benefícios são inatingíveis se utilizado outros tipos de leites (Cruz; Sebastião, 2015).

Segundo o Ministério da Saúde (2005), os RN prematuros podem ser definidos como bebês que nasceram antes das 37 semanas de gestação. Essa definição possui variação se perguntada diretamente para as mães dessas crianças, recebendo respostas com fundo amoroso, como dizer que é fofo e pequeno, sem perder a centralidade de entender que é uma criança que requer cuidados extra, mas sem possuírem o conhecimento da imaturidade dos sistemas, sucedida dessa prematuridade (Bezerra et al., 2017).

O prematuro deve receber como primeira e se possível, única escolha, o leite materno, pois este é suficiente para as necessidades do bebê, possuindo ainda, várias qualidades imunológicas, que irão auxiliar no crescimento, desenvolvimento e na imaturidade (Balaminut et al., 2018).

Em relação a essa qualidades, Zulin et al (2015), apresenta o conhecimento materno, elas reconhecem a importância da amamentação para o desenvolvimento infantil, imunidade. Em contrapartida a isso, apesar de sabermos que existe grande influência do aleitamento materno na prematuridade e na diminuição de doenças ou problemas de saúde, ainda é um tema pouco debatido em nosso âmbito social e em pesquisas, o que pode dificultar o acesso a informações para as mães (Bonilha et al., 2017).

A teoria difere-se do que acontece na realidade, a pesquisa de Nunes et al (2017) comprova isso ao apresentar dados nos quais nenhuma mãe pode amamentar o filho recém-nascido prematuro em seguida ao parto, então, a imaturidade somada a diferenciação de experiências dos cuidados perinatais podem ser fortes fatores para influenciar diretamente um possível retardamento do desenvolvimento neuropsicomotor da criança.

Em contrapartida, um estudo realizado por Pachu e Viana (2018) narram o oposto ao que foi apresentado anteriormente, onde mais de 70\% dos RN da unidade estavam recebendo o aleitamento materno exclusivo. Após a alta, ambiciona-se que desses RN que estavam mamando durante a internação, mais de $80 \%$ prossigam no aleitamento (Luz et al., 2018).

Congiu et al (2019) evidencia dados relevantes sobre o leite de mães com recém nascidos prematuros, apresentando-se com diferença significativa no conteúdo de proteínas e no conteúdo da lactose. Esse fator ocorre provocando um aumento do seu conteúdo total, como uma forma de compensação da imaturidade apresentada pelo lactente. A criança deve ser constantemente avaliada ao nascer, observando e acompanhando o gráfico de peso e crescimento, pois nos primeiros dias ocorre um declínio do peso, principalmente nas em amamentação exclusiva. Esse processo é natural e fisiológico, devendo atentar se foi reestabelecido o peso inicial em torno do $5^{\circ}$ dia. Só deve ser considerado preocupando se a ingestão for muito baixa, resultando em grande perda de peso e complicações no desenvolvimento neuropsicomotor (Ministério da Saúde, 2009 \& 
Tonkin et al., 2018).

Seguindo a mesma linha raciocínio, de maneira geral, esse fator também é observado nos ganhos de peso e comprimento em bebês que se encontram em leite materno exclusivo, Toftlund et al (2018) apresenta em sua pesquisa dados de que crianças que amamentaram, apresentaram maior crescimento e peso entre a faixa etária de 3 a 6 anos. Da mesma forma que, em prematuros também observa-se esses ganhos, principalmente pela busca de compensar o quão imaturo o fisiológico se encontra (Halleux et al., 2019 \& Marino et al., 2019).

Além da parte física, pode-se citar como um dos benefício da amamentação, uma melhor microestrutura da substância branca do cérebro. Essa substância branca do cérebro pode ser definida como fibras complexas e organizadas, que atuam diretamente nos circuitos neurais e garantem o pleno funcionamento do sistema nervoso central (Blesa et al., 2019 \& Dini et al., 2009).

Também dentre os benefício, Fonseca et al (2018) corrobora a diminuição do intervalo de internações e a diminuição do risco dos RN, principalmente os prematuros, virem a desenvolver a sepse. A sepse pode ser definida como uma síndrome de resposta inflamatória sistêmica, ocasionada por uma infecção que gera uma resposta inesperada e complexa (Santos et al., 2019).

Entretanto, a pesquisa de McKinney et al (2019) transparece dificuldades enfrentadas para poder usufruir desses benefícios, como a amamentação exclusiva não ser frequentemente alcançada. Nandakumar et al (2020) alude maior dificuldade em mães de prematuros, devido a imaturação levar a dificuldade na sucção do leite, provando retardo na ativação secretora da mama.

Os benefícios advindos com a amamentação são diversos, mas esse processo vem acompanhado de empecilhos, sendo assim, deve ser facilitado pelos profissionais de saúde, buscando realizar orientações, educação em saúde e apoio para essas mulheres. Confirmando tal importância, Ericson e Palmér (2019) mencionam na sua pesquisa, que as mães exteriorizaram e manifestaram sensibilidade ao engajamento dos profissionais de saúde.

Guerra et al (2021) aborda os aspectos negativos, apresentando dados em sua pesquisa que 30\% das mulheres não pretendiam amamentar mais do que os 6 meses, em decorrência de experiências ruins ou da inclusão da mulher no mercado de trabalho, acarretando na volta após a licença maternidade. Ele abordou ainda dados de que $40 \%$ das mulheres não receberem orientações durante as consultas de pré-natal, ocasionando em maiores números de mães que abandonam o aleitamento materno.

A participação dos profissionais, através do processo de escuta qualificada e de orientações, influenciam diretamente o avançar positivo da amamentação exclusiva, devendo apoia-las em todas as etapas, iniciando desde a decisão de ter um bebê até o puerpério e as consultas de puericultura. Entretanto, tal ação só é possível se houver cooperação de toda a equipe multiprofissional da saúde, para que então, possa ser realizado um acompanhamento digno e completo, atuando perante cada necessidade especifica (Vieira et al., 2020).

\section{Conclusão}

Conforme o que foi abordado durante essa revisão, conclui-se que a amamentação proporciona diversos benefícios para os recém nascidos, principalmente os prematuros, pois auxilia na superação de imaturidade, no crescimento e no desenvolvimento físico, motor e cognitivo.

Todavia, observou-se também que esse processo carece de muita atenção e trabalho da mãe e dos profissionais da saúde, pois ele pode vir acompanhado de dificuldades, como pega incorreta, dificuldade de sucção, lesões nas mamas, gerando empecilho para o aleitamento materno exclusivo.

Dessa forma, entende-se que a amamentação deve ser muito bem orientada e estimulada para a sua implementação, 
pois acarreta benefícios para a mãe e para o bebê, auxiliando na bem-estar e na saúde física e mental dos dois.

Sugere-se para trabalhos futuros, o desenvolvimento de pesquisas em campo, podendo abordar a opinião da equipe de saúde, abordar a vivência e os aspectos da amamentação sob o olhar materno, abordar as dificuldades enfrentadas por mulheres para amamentar, principalmente os prematuros, dentre outros.

\section{Referências}

Alves, J. S., Oliveira, M. I. C., \& Rito, R. V. V. F. (2018). Orientações sobre amamentação na atenção básica de saúde e associação com o aleitamento materno exclusivo. Ciência \& Saúde Coletiva, 23(4), 1077-1088,

Balaminut, T., Sousa, M. I., Gomes, A. L. M., Christoffel, M. M., Leite, A. M., \& Scochi, C. G. S. (2018). Aleitamento materno em prematuros egressos de hospitais amigos da criança do sudeste brasileiro. Revista Eletrônica de Enfermagem.

Bezerra, M. J., Carvalho, A. C. O., Sampaio, K. J. A. J., Damasceno, S. S., Oliveira, D. R., \& Figueiredo, M. F. E. R. (2017). Percepção de mães de recémnascidos prematuros hospitalizados acerca da amamentação. Rev. baiana enferm. 31(2).

Blesa, M., Sullivan, G., Anblagan, D., Telford, E. J., Quigley, A. J., Sparrow, S. A., Serag, A., Semple, S. I., Bastin, M. E., \& Boardman, J. P. (2019). Early breast milk exposure modifies brain connectivity in preterm infants. Neuroimage, 184, 431-439.

Bonilha, A. L. L., Teles, J. M., Tronco, C. S., \& Porciúncula, M. B. (2017). Ações para a promoção do aleitamento materno na prematuridade tardia. Revista Eletrônica Acervo Saúde, 9(3).

Brasil. Ministério da Saúde, Secretaria de Atenção à Saúde, Departamento de ações programáticas estratégicas. Agenda de Compromissos para a Saúde Integral da Criança e Redução da Mortalidade Infantil. Série a. Normas e Manuais Técnicos: Brasília df; 2005

Brasil, Ministério da Saúde. Departamento de Atenção Básica. Aleitamento Materno e Alimentação Complementar, Cadernos de Atenção Básica $n^{\circ}$ 23, $2^{\text {a }}$ edição, 2015.

Congiu, M., Reali, A., Deidda, F., Dessì, A., Bardanzellu, F., \& Fanos, V. (2019). Breast Milk for Preterm Multiples: More Proteins, Less Lactose. Twin Res Hum Genet, 22(4), 265-271.

Cruz, M. R., \& Sebastião, L. T. (2015). Amamentação em prematuros: conhecimentos, sentimentos e vivências das mães. Distúrbios Comun. 27(1), 76-84.

Dini, L. I., Vedolin, L., Aesse, F., Pinheiro, C. P., Luersen, G. F., Wagner, F. V., Isolan, G. R, Mazzola, A., \& Costa, J. C. (2009). Estudo anatômico da substância branca do cérebro: da técnica de Klingler à dissecção virtual por ressonância magnética (tratografia). Jornal Brasileiro de Neurocirurgia, 20(1), 1021 .

Ericson, J., \& Palmér, L. (2019). Mothers of preterm infants' experiences of breastfeeding support in the first 12 months after birth: A qualitative study. Birth, 46(1), 129-136, 2019.

Fonseca, L. T., Senna, D. C., Eckert, G. U., Silveira, R. C., \& Procianoy, R. S. (2018). Association between human breast milk and retinopathy of prematurity. Arq. bras. Oftalmol., 81(2), 102-109.

Gomes, T. M. V., Soares, C. B., Silva, A. R., Ferreira, D. S., Silva, N. R., Sales, M. C., \& Sousa, I. M. (2020). Fatores relacionados à prematuridade em uma maternidade pública de Teresina - PI: estudo retrospectivo. Rev. Pesqui. Fisioter., 10(1), 69-76.

Gonçalves, J. R. Como escrever um artigo de revisão de literatura. Revista JRG de Estudos Acadêmicos, 2(5).

Guerra, B. C. O., Silva, L. R., Christoffel, M. M., Monnerat, I. C., Silva, L. J., Teixeira, S. V. B, Santos, I. M. M., Zuzarte, J. S., \& Estrela, A. C. G. S. (2021). A avaliação da autoeficácia de nutrizes em amamentar para o cuidado de enfermagem. Research, Society and Development, 10(1),

Halleux, V., Pieltain, C., Senterre, T., Studzinski, F., Kessen, C., Rigo, V., \& Rigo, J. (2019). Growth Benefits of Own Mother's Milk in Preterm Infants Fed Daily Individualized Fortified Human Milk. Nutrients, 11(4)

Luz, L. S., Minamisava, R., Scochi, C. G. S., Salge, A. K. M., Ribeiro, L. M., \& Castral, T. C. (2018). Predictive factors of the interruption of exclusive breastfeeding in premature infants: a prospective cohort. Rev Bras Enferm, 71(6), 2876-2882.

Marino, L. V., Fudge, C., Pearson, F., \& Johnson, M. J. (2019). Home use of breast milk fortifier to promote postdischarge growth and breast feeding in preterm infants: a quality improvement project. Arch Dis Child, 104(10), 1007-1012.

McKinney, C. M., Plange-Rhule, G., Ansong, D., Cunningham, M. L., Agyeman, I., \& Coffey, P. S. (2019). A randomized crossover trial comparing the Nifty cup to a medicine cup in preterm infants who have difficulty breastfeeding at Komfo Anokye Teaching Hospital (KATH) in Kumasi, Ghana. PLoS One, 14(10).

Monteiro, J. R. S., Dutra, T. A., Tenório, M. C. S., Silva, D. A. V., Mello, C. S., \& Oliveira, A. C. M. (2020). Fatores associados à interrupção precoce do aleitamento materno exclusivo em prematuros. Arq. Catarin Med., 49(1), 50-65.

Nandakumar, A., Pournami, F., Prabhakar, J., Nair, P. M. C., \& Jain, N. (2020). Exclusive Breast Milk vs. Hybrid Milk Feeding for Preterm Babies-A Randomized Controlled Trial Comparing Time to Full Feeds. J Trop Pediatr, 66(1), 38-45.

Nunes, C. R. N., Campos, L. G., Lucena, A. M., Pereira, J. M., Costa, P. R., Lima, F. A. F., \& Azevedo, V. M. G. O. (2017). Relação da duração da posição canguru e interação mãe-filho pré-termo na alta hospitalar. Revista paul. Pediatr, 35(2), 136-143. 
Research, Society and Development, v. 10, n. 4, e13110413839, 2021

(CC BY 4.0) | ISSN 2525-3409 | DOI: http://dx.doi.org/10.33448/rsd-v10i4.13839

Pachu, H. A. F., \& Viana, L. C. (2018). Aleitamento materno em uti neonatal. Revista Nova Esperança, 16(2), 58-65.

Pereira, A. S., Shitsuka, D. M., Parreira, F. J., Shitsuka, R. Metodologia da Pesquisa Científica, Universidade Federal de Santa Maria.

Rocha, E. M. A., Macedo, L. K. M., Borges, L. V. A., Pinheiro, A. M. C., Santos, R. S, Conceição, H. N., \& Câmara, J. T. (2020). Breastfeeding, peaceful and pleasant breastfeeding: an experience report. Research, Society and Development, 9(7), 1-8.

Rosa, N. P., Oliveira, D. C., Jantsch, L. B., \& Neves, E. T. (2020). Moderate and late previous pregnant baby health accidents in the neonatal period. Research, Society and Development, $9(7), 1-8$.

Santos, M. R., Cunha, C. C., Ishitani, L, H., \& França, E. B. (2019). Mortes por sepse: causas básicas do óbito após investigação em 60 municípios do Brasil em 2017. Revista Brasileira de Epidemiologia, 22(3).

Spezzia, S. Maloclusão e prematuridade ao nascimento. Journal of Oral Investigations, 9(1), 67-81.

Toftlund, L. H., Halken, S., Agertoft, L., \& Zachariassen, G. (2018). Catch-Up Growth, Rapid Weight Growth, and Continuous Growth from Birth to 6 Years of Age in Very-Preterm-Born Children. Neonatology, 114(4), 285-293.

Tonkin, E., Miller, J., Makrides, M., McPhee, A. J., Morris, S. A., Gibson, R. A., \& Collins, C. T. (2018). Dietary Protein Intake, Breast Feeding and Growth in Human Milk Fed Preterm Infants. Int J Environ Res Public Health, 15(6).

Vieira, C. M., Freitas, H. M. B., Zanon, B. P., \& Anversa, E. T. R. (2020). Promoção do aleitamento materno exclusivo na visão dos profissionais de uma Estratégia Saúde da Família. Research, Society and Development, 9(8).

Vitolo, M. R. Nutrição - da Gestação ao Envelhecimento, Rubio Editora, (2a ed.).

Zulin, N. E., Tacla, M. T. G. M., Souza, S. N. D. H., Monteiro, A. T. A., \& Ferrari, R. A. P. (2015). Vivência de mães de prematuros no processo de translactação. Semina: Ciências Biológicas e da Saúde, 36(1), 363-372. 